\title{
A Personalised Virtual Coach to Counteract Ageing Decline: The H2020 NESTORE Project
}

\author{
Maria Renata Guarneri', Alfonso Mastropietro' ${ }^{2}$, Maurizio Caon ${ }^{3}$, Laura \\ Fernandez Maldonado ${ }^{4}$, Francesco Furfari ${ }^{5}$, Giuseppe Andreoni ${ }^{1,2}$, Giovanna \\ Rizzo $^{2 *}$ on behalf of the NESTORE Consortium
}

\begin{abstract}
Ageing population is growing faster in EU. ICT can provide solutions for Active Ageing; however, the success of novel ICT solutions depends on the user perception of their efficacy to support toward health promotion and global wellness. In this context, the H2020 project NESTORE (Non-intrusive Empowering Solutions and Technologies for Older people to Retain Everyday life activity) will develop an innovative, multidimensional, personalised e-coaching system to support healthy ageing by: 1) Generating and sustaining motivation to take care of health; 2) Suggesting healthy nutrition and personalised physical and mental coaching, as well as social interaction, to prevent decline and preserve wellbeing. NESTORE started in September 2017 and will last three years. It involves 16 partners from 7 European countries.
\end{abstract}

Keywords Ageing $\cdot$ Virtual Coach $\cdot$ ICT

\footnotetext{
${ }^{1}$ MR. Guarneri, G. Andreoni

Politecnico di Milano, Dipartimento di Design, Milano, Italy.

e-mail: mariarenata.guarneri@polimi.it; giuseppe.andreoni@polimi.it
}

${ }^{2}$ A. Mastropietro, G. Rizzo

Istituto di Bioimmagini e Fisiologia Molecolare, Consiglio Nazionale delle Ricerche, Segrate, Italy.

e-mail: alfonso.mastropietro@ibfm.cnr.it; giovanna.rizzo@ibfm.cnr.it

${ }^{3}$ M. Caon

University of Applied Sciences and Arts Western Switzerland, Fribourg, Switzerland

e-mail: maurizio.caon@hes-so.ch

${ }^{4}$ L. Fernandez

Institut de l'Envelliment, Universitat Autonoma de Barcelona, Barcelona, Spain

e-mail: laura.fernandezm@uab.cat

${ }^{5}$ F. Furfari

Istituto di Scienza e Tecnologie dell'Informazione "Alessandro Faedo", Pisa, Italy

e-mail: Francesco.furfari@isti.cnr.it

*corresponding author 


\section{Introduction}

Due to longer life expectancy and the declining fertility rates, the proportion of people aged over 65 years is growing faster in most of the developed countries. Some forecasts suggest that the population of elderly people will almost double from 87.5 million in 2010 to 152.6 million in 2060 [1].

The emerging social aspect related to ageing population introduces some crucial challenges to society and healthcare systems. Without adequate adjustments, i.e. social, economic and demographic policies as well as changes in people's behaviours, the process can trigger certain negative consequences in the long term. In order to maximise the wellbeing of older people and to reduce the economic burden of their care, the health systems should promote "healthy ageing" as much as possible. Healthy ageing is a common word that represents a complex intervention because it tackles all the human domains: physical, metabolic, cognitive, social, etc. Thus, a multidomain, multifactorial system to promote proper healthy strategies has to be designed and NESTORE project aims at this integrated vision as we will explain below.

Furthermore, promoting healthy ageing could represent a strategic opportunity for the economic growth thanks to the higher spending capacity of older people (over $€ 3.000$ billion for the population $<65$ ). The EU has focused its attention on the concept of "Silver Economy" which is driven by the rise of new consumer markets and by the need to improve the sustainability of public expenditure linked to ageing.

In this direction, the rapid development of the ICT, and in particular mobile technologies, offers an important opportunity for addressing the development of integrated solutions to support active and healthy ageing. The availability of new integrated ICT technologies and its widespread diffusion could contribute dramatically to the implementation of a sustainable silver economy. Moreover, ICT solutions allow, in an innovative manner, introducing the possibility of a new technological framework to re-design the healthcare system model.

In this context, the project NESTORE (Novel Empowering Solutions and Technologies for Older people to Retain Everyday life activities), funded by EU H2020 programme, was designed and developed [2].

The project aims at the development of an integrated solution, based on nonobtrusive interaction with the person, which will support healthy older people to sustain their healthy life by promoting personalised pathways to wellbeing. The personalised support will be provided by an intelligent e-coaching system, which leveraging monitoring technologies, social connectivity and gamification mechanics are able to support older people to maintain independence by encouraging them to become co-producers of their wellness (patient empowerment) by:

- generating self-awareness (understanding risks associated with ageing),

- enhancing and sustaining motivation to take care of their health (solutions that support healthy lifestyles and are able to evolve as the person ages) 
- $\quad$ support the healthy ageing process based on healthy nutrition and adequate physical and mental activity/exercise.

\section{The wellbeing dimensions of NESTORE}

Ageing is a multi-domain process. It involves the social, economic, physical, psychological and mental spheres and all these domains are strongly interconnected. Some crucial factors that affect the wellbeing and the quality of life of the subjects are the nutritional habits, physical activity, mental health and social capital.

Good nutrition plays a significant role in the wellbeing of healthy older people and in delaying and reducing the risk of contracting diseases. General dietary recommendations on what should be eaten are relevant and important for older people. They should, for example, not choose food that has a high salt or sugar content; they should choose soft fats instead of hard fats, choose food with good carbohydrates and large amounts of fibre, and prepare their food with as little cooking fat as possible. Other important dietary components include vitamin D and calcium, especially considering the high prevalence of osteoporosis and hip fractures.

Ageing can be related to psychological and cognitive decline [3]. Mental Health was defined by the WHO as "a state of wellbeing in which the individual realises his or her own abilities, can cope with the normal stresses of life, can work productively and fruitfully, and is able to make a contribution to his or her community". In order to ensure a mentally healthy status, some interventions should be provided considering many aspects, such as participation in meaningful activities, strong personal relationships, physical health.

Ageing is characterised, among other things, by motor function impairment such as coordination difficulty, increased variability of movement, slowing of movement, and difficulties with balance and gait in comparison to young adults [4]. These deficits have a negative impact on the ability of older adults to perform activities of daily living and may result in social exclusion as well as in an increased risk of traumatic events (i.e. falls). Even if dysfunctions of the central and peripheral nervous systems are not involved, progressive and generalised loss of skeletal muscle mass and strength (sarcopenia) may play an important role [4]. Physical activity is believed to be the most effective of all interventions proposed to counteract skeletal muscle impairment in older adults, improving quality of life and functionality $[5,6]$.

Another important aspect is the preservation of the social capital provided by older people. The socio-economic status, social integration and high personal competencies are related to higher subjective wellbeing. The quality of the social contacts is, as well, an important factor to consider for a better wellbeing [7]. Moreover, the voluntary work, as well as the educational and social activity group interventions, can improve the mental health and prevent social isolation and loneliness among the older people. 


\section{Scope of NESTORE and key innovations}

NESTORE proposes the development of a multi-dimensional system that covers all main domains affected by the ageing process, able to provide services, applications and a rewarding system to support active and healthy ageing with a user-centred approach.

While leveraging the state-of-the-art technologies, the innovation brought about by NESTORE relies, first, on its integration capacity. We can see NESTORE according to different perspectives:

1. as a platform, providing a set of basic services that can be leveraged by different apps, potentially offered by different stakeholders;

2. as a companion, offering personal and personalised support according to the user needs and interests;

3. as a social support, easing communication with family and friends as well as with carers, but also able to propose external service offerings based on "group" interests.

The main outcome of NESTORE is an e-coaching system [8], which may be implemented through a dedicated mobile App and also embodied in a smart object offering tangible interfaces in relation to the user's preferences. This e-coach will suggest and guide the user toward different "wellbeing pathways" providing tailored interventions exploiting personalised preferences and capabilities. The target population will include "fit older people" (i.e., good physical performance and low risk of near-future disabilities).

Key innovation element is the development of a multi-domain "cloud-based intelligence" able to interpret different signals coming from an extensive sensing system, to provide feedback that is meaningful, timely and relevant to the user. The effectiveness of the feedback will be assured by the adoption of well-assessed psychological developmental regulation and health behaviour change models, such as the SOC model [9] and the Health HAPA approach [10]. Emotion analysis and recognition, emotional computing, tangible and affective interfaces, will be used to ensure user engagement with the e-coaching system. According to such models, we have developed the concept of "pathways of interest" able to provide hints, suggestions and services according to the user's elective preferences while ensuring that all the five dimensions of wellbeing are satisfied to maximise the overall health status.

The coaching on the different pathways will be supported by different techniques, leveraging multimodal communication in order to provide an engaging and playful user experience: 1) natural dialogue with the user; 2) tangible interfaces for self-reflection and behaviour change; 3) social and environmental support through the social platform; 4) serious games and gamification; 5) apps for self-monitoring. 
From the user point of view, NESTORE can be seen according to two different perspectives: friend and coach.

As a friend, NESTORE fully understands the emotional status and the wishes of the user; it is able to establish a personal and long-lasting relationship with the user interacting in a natural way.

As a coach, NESTORE understands the "weaknesses" of the user and proposes actions and activities that compensate shortcomings, re-establishing a status of wellbeing and maintaining such status in time. NESTORE is a ubiquitous conversational agent acting as a caring coach that will interact with the user assuming many forms, will use different modalities (voice, text, apps, tangible interfaces), and will be adapted to the user's preferences and to the contextual information.

In order to ensure user acceptance of the e-coaching system, co-design and participatory approaches will be adopted and used throughout the project duration. Such tools and methods will seek to engage and elicit information related to perception, acceptance and usability of technology to support healthcare and wellbeing.

\section{The NESTORE methodological approach and validation}

The NESTORE scientific and technical approach is modelled around two guiding principles: i) combining agility and structure and ii), adopting Co-Design and UCD (User-Centred Design) as methodological approaches for the overall definition of the NESTORE solution [11]

i. $\quad$ Agility and structure. NESTORE is driven by a combination of Agile Development methods and techniques with the structure necessary to a large and distributed collaboration effort.

ii. $\quad$ Adoption of Co-Design and UCD. NESTORE methodology incorporates the overarching principle of having users as active, participating actors in the project in the typical approach of UCD.

This general approach has been translated into the following strategic planning choices:

1. The iterative process structured along major milestones is enriched by intermediate working releases (with varying, incremental set of features);

2. The system architecture development is linked to the integration work in which all the different modules and elements are made available for technical and users' short or long-time evaluations. The NESTORE Integration strategy is entrusted also with the mission of maintaining a unifying technology view;

3. The user point of view is championed and maintained through a co-design approach that will be adopted for the definition of the system requirements and for the user testing that will be performed by means of small-scale pilots; 
4. Agility steers the work by avoiding a once-in-a-time, "big design upfront" effort, and making instead service design a continuous task in the project lifetime, to guarantee that technological innovations and user feedback are effectively translated in the NESTORE features;

5. Scenarios and requirements will be used as flexible design tools. From the business and end-users point of view, NESTORE will work with mock-ups and working prototypes.

The strategic planning choices listed above strongly shape the scientific and technical approach followed by the project. The structure of the project has been designed to satisfy the above principles with technical work-packages working in close cooperation, guided by a set of user-based requirements that are implemented in an incremental manner and verified with the users in a continuous cycle. The NESTORE structure has been organised according to the workflow described and depicted below:

Requirements Definition, for defining the functional requirements taking into accounts the ageing process.

Iterative Design of NESTORE Solution, for providing the user point of view and refine requirements according to a co-design methodology.

This will guide the:

Development of NESTORE platform and components: this work is organised according to three lines of research: i) the "passive" sensing system based on wearable sensor technologies and environmental sensors, ii) the interpretation and reasoning algorithms, and iii) the user interface at large, the interaction with the virtual coach and the engagement of the users via a social platform as well as series of serious games. The above components will be brought together through the

Integration of the NESTORE System that will provide the overall technical solution for the provision of the user services (see Fig.1),

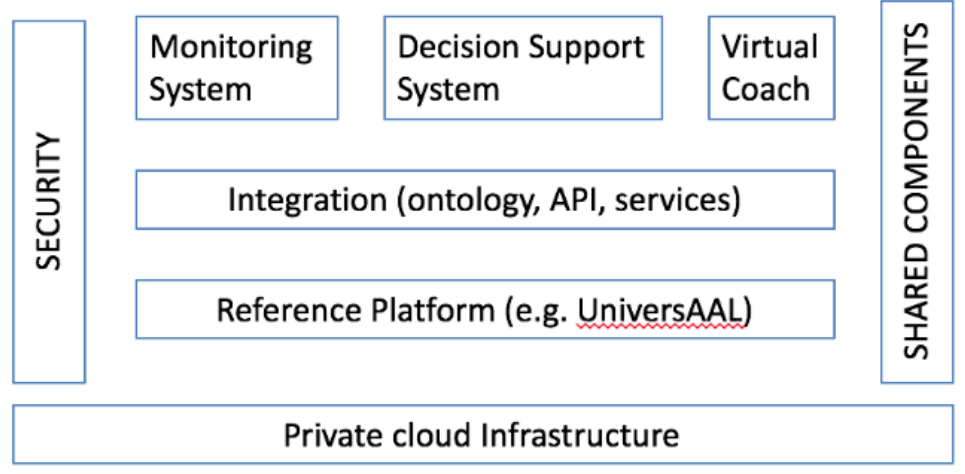

Fig 1. Integration architecture. 
Evaluation and User Feedback: this phase will generate insights about the service value from the user point of view and will feedback in a new updated loop. The integrated system will be tested in three pilot sites (Italy, Spain and The Netherland).

The piloting test will evaluate the usability, sensors and devices functionality, and overall user acceptance. In particular, experience of the participants with the system will be evaluated, considering different aspects such as: quality of technology (efficiency, effectiveness, usefulness, completeness and accuracy), reliability of the system (reliability, security, interoperability and ease of repair and maintenance), usability and acceptance (ease of use, graphic design acceptance, user satisfaction, navigation, user control, time, user experience, mental effort, support, learning and acceptance), use of monitoring (frequency, modus operandi, user profile, context in the platform's use), problem solving (useful alerts, false alarms, emergency calls, quality life improvement, isolation problems solution, cognitive stimulation and physical activity motivation).

In the pilots, the efficacy of the system will also be evaluated in each one of the wellbeing domains (physical-physiological, nutritional, cognitive, mental, social) included in NESTORE: validated clinical measurements, like additional questionnaires, medical imaging and signals, clinical geriatric evaluation in supervised sessions, will be considered to provide quantitative metrics of validations on:

- Physiological status and physical activity behaviour (Sedentariness, muscle strength and cardiorespiratory fitness, sleep quality, and active physical behaviour);

- Nutritional behaviour (quality of diet and quantity of food)

- Cognitive function (memory, processing speed, reaction time, verbal fluency)

- Mental status and social behaviour (depressive symptoms, life satisfaction, social relation and integration).

Some complementary measurements will help to provide information on possible positive effects on the body produced by using the NESTORE platform.

Moreover, techno-economics will be evaluated, as well as the feasibility of different organisational and business models aimed at achieving the sustainability of the NESTORE solution.

\section{Cost-effectiveness and sustainability of NESTORE}

Past literature pointed out that the interventions addressed to increase the awareness about physical-physiological, nutritional, cognitive, mental and social conditions of $60+$ citizens are cost-effective from the national healthcare system perspective: indeed, they allow, for instance, to reduce the number of avoidable hospitalisations and drug consumption as well as to improve the management of chronic diseases and citizens' quality of life.

According to this perspective, the Pilots will gather evidence on the fact that the NESTORE technological solutions enable citizens/users to improve their conditions 
(physical, cognitive, mental and social) as well as their behaviours (e.g. eating habits, etc.). With regard to the effectiveness, the focus will be also on the wellbeing as well as on users' empowerment, health literacy and capability of self-care. Indeed, regardless of the IT literacy, NESTORE is expected to improve all these aspects.

The necessary condition for generating cost-effective impacts on the system will be the effective usage of NESTORE solutions by the citizens/users.

Past studies highlighted that most of the technological solutions aimed at maintaining lifestyle and supporting self-care and care management are not used regularly by users/citizens and thus their results fall far behind their promises or what measured in 'Lab' contexts. The main reasons, as known, are two: i) they are not considered relevant/useful, ii) they are not easy to use.

Considering this, the project will allow to test and assess with final users the perceived usefulness and the ease of use of the different NESTORE solutions in order to gather evidence on their actual usage; moreover, through qualitative and co-design approaches will be analysed the willingness of citizens to adopt these solutions in the long-term.

All data collected by the Pilots and during the co-design process will permit to assess the potential cost-effectiveness of NESTORE and its technological solutions compared to the current practices by means of a control group.

\section{Conclusions}

Within NESTORE, we attempt to support healthy ageing with an integrated, usercentred and non-obtrusive approach, tested in real-life environments, in order to achieve wide acceptance by the target users as well as to accelerate the digital innovation process. Therefore, we believe that this project has the potential of delivering sustainable innovation in the area of active and healthy ageing. Currently, the User's analysis was run and the matching among requirements from users, technology, and expert is running to provide the first version of the platform design.

\section{Acknowledgments}

NESTORE is funded by the European Commission under the H2020 programme, GA769643. The authors wish to thank all the project partners for their contribution to the project.

The authors want to thank the NESTORE Consortium and in particular: Leonardo Angelini, Elena Mugellini (University of Applied Sciences and Arts of Western Switzerland, Switzerland); Simone Porcelli, Filippo Palumbo, Michele Girolami (Consiglio Nazionale delle Ricerche, Italy); Christina Roecke (University of Zurich, Switzerland); Josep Del Bas, Silvia Orte, Sebastian Idelsohn, Filip Velickovski (Fundació Eurecat, Spain); Martin Sykora (Loughborough University, United Kingdom); Petia Radeva (University of Barcellona, Spain); Emanuele Lettieri, Paolo Bertelè (Politecnico di Milano, Italy); Antoni Salvà (Fundació Salut i 
Envelliment, Spain); Estelle Huchet (AGE, Belgium); Giuseppe Baroni, Corrado Galzio, (Flextronics Design Srl, Italy); Ciprian Candea, Gabriela Candea (ROPARDO Srl, Romania); Paul Chamberlein, Claire Craig (Sheffield Hallam University, United Kingdom), Stephan Lukosch (Technische Universiteit Delft, The Netherlands).

\section{References}

1. Population ageing in Europe: facts, implications and policies. (2014) https://ec.europa.eu/research/social-sciences/pdf/policy_reviews/kina26426enc.pdf

2. NESTORE Official Web-Site https://nestore.ro/home

3. Klimova B, Valis M, Kuca K. Cognitive decline in normal aging and its prevention: are view on non-pharmacological lifestyle strategies. Clin Interv Aging. 2017 May 25;12:903-910.

4. Di Tano G, Fulle S, Pietrangelo T, Bellomo R, Fanò G. Sarcopenia: characteristics, genesis, remedies. Sport Sci Health. 2005;1(2):69-74.

5. Nelson ME, Rejeski WJ, Blair SN, et al. Physical activity and public health in older adults: recommendation from the American College of Sports Medicine and the American Heart Association. Med Sci Sports Exerc. 2007;39(8):1435-1445.

6. Freiberger E, Sieber C, Pfeifer K. Physical activity, exercise, and sarcopenia - future challenges. Wien Med Wochenschr. 2011;161(17-18):416-425.

7. Charles S, Carstensen LL. Social and Emotional Aging. Annu Rev Psychol. 2010; 61: 383 409.

8. Kamphorst BA. E-coaching systems. Personal and Ubiquitous Computing. 2017 Aug $1 ; 21(4): 625-32$.

9. Freund, A. M., \& Baltes, P. B. (1998). Selection, optimization, and compensation as strategies of life management: correlations with subjective indicators of successful aging. Psychology and aging, 13(4), 531.

10. Schwarzer, R., Lippke, S., \& Luszczynska, A. (2011). Mechanisms of health behavior change in persons with chronic illness or disability: the Health Action Process Approach (HAPA). Rehabilitation psychology, 56(3), 161

11. Beyer H, Holtzblatt K, Baker L. An Agile customer Centred Method: Rapid Contextual Design. 2004; Lecture Notes in Computer Sciences, Springer. 